\title{
INTEGRABILITY FOR RELATIVISTIC SPIN NETWORKS
}

\author{
JOHN C. BAEZ AND JOHN W. BARRETT
}

\begin{abstract}
The evaluation of relativistic spin networks plays a fundamental role in the Barrett-Crane state sum model of Lorentzian quantum gravity in 4 dimensions. A relativistic spin network is a graph labelled by unitary irreducible representations of the Lorentz group appearing in the direct integral decomposition of the space of $L^{2}$ functions on three-dimensional hyperbolic space. To 'evaluate' such a spin network we must do an integral; if this integral converges we say the spin network is 'integrable'. Here we show that a large class of relativistic spin networks are integrable, including any whose underlying graph is the 4-simplex (the complete graph on 5 vertices). This proves a conjecture of Barrett and Crane, whose validity is required for the convergence of their state sum model.
\end{abstract}

\section{INTRODUCTION}

In formulating a state sum model for 4-dimensional Lorentzian quantum gravity, Barrett and Crane [11] used the notion of a 'relativistic spin network', which is simply a graph with edges labelled by nonnegative real numbers. These numbers parametrize a certain class of irreducible unitary representations of the Lorentz group. The model involves a triangulation of spacetime, and for each 4-simplex one must calculate an amplitude associated to a relativistic spin network whose underlying graph is the complete graph on 5 vertices:

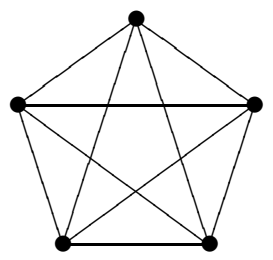

To calculate this amplitude one must do an integral. In [11 this integral was conjectured, but not proven, to converge. Here we prove that it does in fact converge. More generally, one can associate a similar integral to any relativistic spin network. To 'evaluate' a relativistic spin network one must do this integral. We prove that this integral 
converges whenever the underlying graph of the spin network lies in a certain large class. This class of graphs includes the tetrahedron and all graphs obtained from it by repeatedly adding an extra vertex connected by at least 3 edges to the existing graph. Thus in particular this class includes the complete graph on 5 vertices.

The results of the paper are formulated in Section 2 and proved in Section 3. These sections are developed in a self-contained manner, so that the reader interested in the mathematical details can simply start there.

The remainder of the introduction contains some background material placing the results in their mathematical and physical contexts. The mathematical part of the introduction explains how the integrals we consider arise from the representation theory of the Lorentz group. The physics part of the introduction sketches how this representation theory has been used in constructing models of fundamental physics.

1.1. Mathematical context. Calculations in the representation theory of compact Lie groups are conveniently expressed in terms of diagrams. Let $a, b, \ldots, n$ and $p, q, \ldots, z$ be representations of the group and $A: a \otimes b \otimes \ldots \otimes n \rightarrow p \otimes \ldots \otimes z$ an intertwining operator (a linear map which commutes with the action of the group). This can be represented by a diagram:

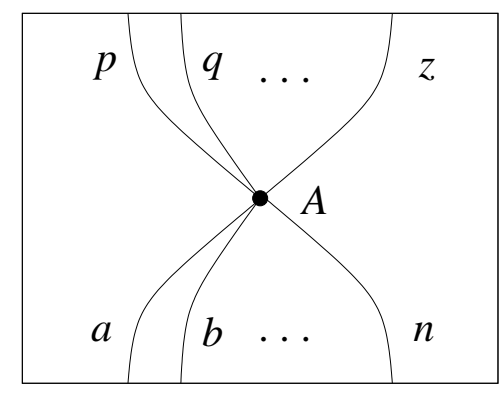

Usually we fix some particular operators and represent them by diagrams like this; these operators are called the elementary operators, or sometimes vertices, corresponding to the fact that the graph in the diagram has just one vertex.

The identity operator is represented by a vertical line with no vertex. Tensoring corresponds to joining diagrams together horizontally, and composition of operators corresponds to joining diagrams vertically. A trace on a representation, say $a=p$ in the above diagram, is represented by joining the edge for $a$ round in a loop to the edge for $p$. 
These processes allow us to build various operators from the elementary ones, with the description in terms of elementary operators being captured by a diagram: a graph with vertices labelled by elementary operators and a certain number of free ends on the bottom and top, corresponding to 'inputs' and 'outputs':

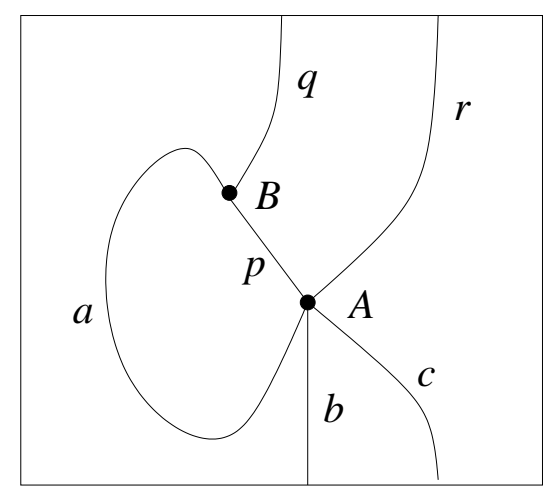

The power of this method comes from the fact that relations between these intertwining operators correspond, in many cases, to deformations of the diagram which can be interpreted as moving the vertices and edges in either two- or three-dimensional space (isotopies). This theory was developed to its fullest extent in the generalisation from compact Lie groups to a certain class of Hopf algebras, particularly the quantum groups. Since a diagram with no free ends corresponds to an intertwining operator from the trivial representation to itself, i.e. essentially just a complex number, such Hopf algebras yield invariants of knots and graphs embedded in three-dimensional space.

However in this paper we are concerned with a generalisation in a different direction, namely from compact to non-compact Lie groups, and study a particular class of unitary representations of the Lorentz group, $\mathrm{SO}_{0}(3,1)$. The subscript here indicates the connected component of $\mathrm{SO}(3,1)$ that contains the identity. This is the group covered by $\operatorname{SL}(2, \mathbb{C})$.

We restrict attention to a particularly simple class of elementary operators (vertices) which are invariant under all permutations of the edges in the diagram. In essence, as will be explained below, the composite intertwining operators depend only on the underlying graph of the diagram and not on the way in which it is drawn on the plane; the knot-theoretic considerations are absent. The difficulties in the theory are of a different nature. The trace cannot be defined in all circumstances since the representations are infinite-dimensional, and 
the ordinary trace of the identity operator is infinite. However we will show that there is another perfectly good notion of trace provided that the diagram is sufficiently connected.

The representations of the Lorentz group considered here are as follows. There is one for each real number $p \geq 0$. The Hilbert space of this representation (also denoted $p$ ) is the space of solutions to the equation $\nabla^{2} f=-\left(p^{2}+1\right) f$ on three-dimensional hyperbolic space, $H$, which have square-integrable boundary data on the sphere at infinity [11. This Hilbert space is characterised by a reproducing kernel on $H$, $K_{p}(x, y)$, which is a smooth, bounded, symmetric integral kernel that solves the equation $\nabla^{2} K=-\left(p^{2}+1\right) K$ in both $x$ and $y$. The formula for $K$ is given in Section 2. The Lorentz group acts by translations on this Hilbert space, and the resulting representation is unitary and irreducible.

The elementary operator $A: p_{1} \otimes \cdots \otimes p_{m} \rightarrow q_{1} \otimes \cdots \otimes q_{n}$ is defined by

(1) $f_{1} \otimes \cdots \otimes f_{m} \mapsto \frac{1}{2 \pi^{2}} \int_{H} K_{q_{1}}\left(y_{1}, z\right) \cdots K_{q_{n}}\left(y_{n}, z\right) f_{1}(z) \cdots f_{m}(z) \mathrm{d} z$

The result of applying the operator $A$ is a function of the variables $y_{1}, \ldots, y_{n}$ in $H$. However it is not clear that this function lies in the Hilbert space $q_{1} \otimes \cdots \otimes q_{n}$. For now, we simply consider the formal expressions that are obtained by composing these operators. We postpone the question about whether these converge to the consideration of closed diagrams (those with no free ends).

The operator $A$ can be thought of as an integral operator, albeit with a distributional integral kernel,

$$
\begin{gathered}
A\left(x_{1}, \ldots, x_{m} ; y_{1}, \ldots, y_{n}\right)= \\
\frac{1}{2 \pi^{2}} \int_{H} K_{q_{1}}\left(y_{1}, z\right) \cdots K_{q_{n}}\left(y_{n}, z\right) \delta\left(z, x_{1}\right) \cdots \delta\left(z, x_{m}\right) \mathrm{d} z
\end{gathered}
$$

with $\delta(x, y)$ the delta function for integration on $H$. With this notation, the composition of operators consists of multiplying their kernels and integrating the common variables over $H$. The trace is naturally expressed using the integral kernels in the same way. For example, the trace of $A$ over the first variable is

$$
\int_{H} A\left(z, x_{2}, \ldots, x_{m} ; z, y_{2}, \ldots, y_{n}\right) \mathrm{d} z .
$$

Combining the elementary operators as described above gives a description of the operator for a general diagram. Since composition and 
trace both involve joining a free end at the bottom of the diagram to a free end on the top, the calculation always gives a factor of

$$
\int_{H} K_{p}\left(y_{1}, x\right) \delta\left(x, y_{2}\right) \mathrm{d} x=K_{p}\left(y_{1}, y_{2}\right)
$$

for each internal edge, with variables $y_{1}, y_{2}$ corresponding to its two vertices.

The operator for a diagram with just two free ends

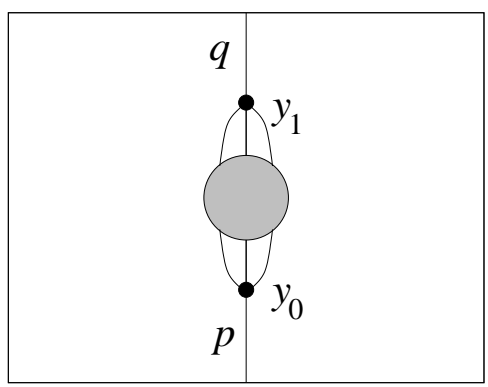

can be described as follows. Associate a variable $y_{i} \in H$ to each vertex in the diagram. Let $E$ be the set of all edges in the interior of the diagram (i.e., not meeting the boundary box). The corresponding operator is

$$
f \mapsto \int \prod_{i} \frac{\mathrm{d} y_{i}}{2 \pi^{2}}\left(\prod_{E} K\right) K_{q}\left(y_{1}, x\right) f\left(y_{0}\right)
$$

In the main body of the paper we consider closed diagrams, those with no free ends on the boundary rectangle. The naive idea for associating a number, or evaluation, to this graph would be to take the trace of the previous graph. But this trace is always infinite. However, by Schur's lemma

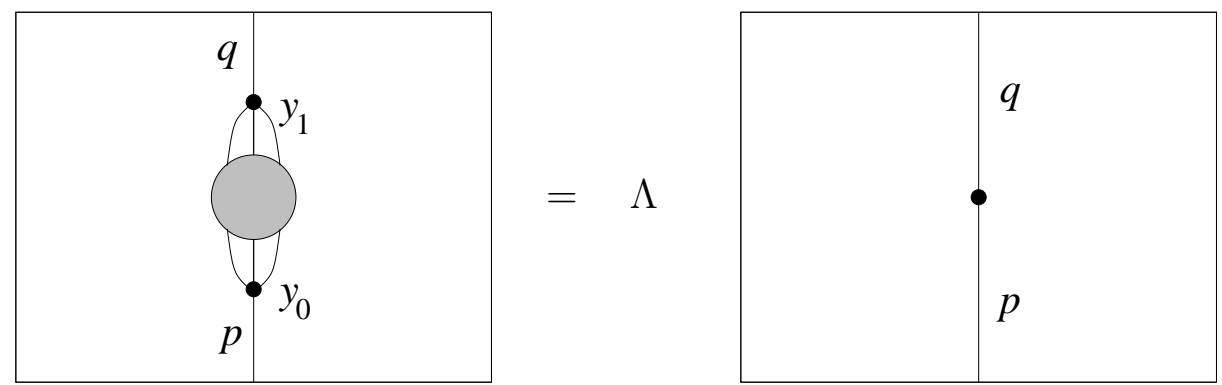

for some constant $\Lambda$, and in fact both sides are proportional to $\delta(p-q)$. 
In what follows, we show that a good definition for the evaluation of the closed diagram

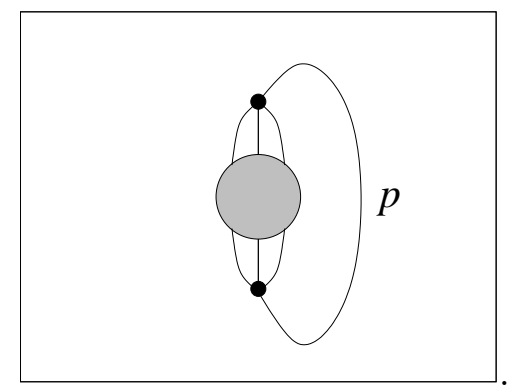

is the above constant $\Lambda$; this constant is given by a multiple integral of products of $K$ 's which converges in many cases. This definition is presented afresh in Section 2 with no reference to the representation theory sketched here. In that section, it is shown that this gives an invariant of the graph which does not depend on the way in which the diagram is drawn in the plane, nor on which edge is chosen to break it into a diagram with two free ends.

1.2. Physics applications. The study of spin networks was initiated by Penrose 21] in the early 1970's, as part of an attempt to a find a description of the geometry of spacetime that takes quantum mechanics into account from the very start. These spin networks were simply graphs with edges labelled by irreducible representations of $\mathrm{SU}(2)$ (i.e. spins $\left.j=0, \frac{1}{2}, 1, \frac{3}{2}, \ldots\right)$ and vertices labelled by intertwining operators. Such spin networks are also implicit in Ponzano and Regge's state sum model of 3-dimensional quantum gravity, published in 1968 [23]. However, this was only realized much later [19].

The real surge of work on spin networks came in the early 1990's, when they were generalized to other groups and even quantum groups. At this point, people began to use them systematically to construct topological quantum field theories. For example, Reshetikhin and Turaev [26] used spin networks to give a purely combinatorial description of Chern-Simons theory and prove that it satisfies the Atiyah axioms for a 3-dimensional TQFT. Shortly thereafter, Turaev and Viro 30 used them to construct a $q$-deformed version of the Ponzano-Regge model and prove that it, too, is a TQFT. We now recognize this theory as a Euclidean signature version of $3 \mathrm{~d}$ quantum gravity with nonzero cosmological constant. Later, Crane and Yetter [13] used spin network technology to construct a state sum model of a 4d TQFT. This appears to be a quantization of $B F$ theory with cosmological constant term.

One reason these topological quantum field theories are interesting is that they share some features with a physically more important but 
also far more problematic theory: 4-dimensional quantum gravity. The success of spin network methods in constructing TQFTs prompted various attempts to apply spin networks to $4 \mathrm{~d}$ quantum gravity. These attempts came from two main directions.

The first was work on 'loop quantum gravity', an approach to the canonical quantization of Einstein's equations. Here Rovelli and Smolin [27] realized that $\mathrm{SU}(2)$ spin networks embedded in a 3-manifold representing space can serve as an explicit basis of kinematical states. Using this basis they showed how to construct operators corresponding to observables such as the areas of surfaces and volumes of regions [28]. This work was soon made rigorous by Ashtekar, Lewandowski, Baez and others, and spin networks quickly became a standard tool in this field [1, 2, 3, 4, 5].

The second direction was work on state sum models of $4 \mathrm{~d}$ quantum gravity. Models of this sort were proposed by Barrett and Crane, first in the Euclidean [10] and then in the Lorentzian signature [11]. Their original models involve a triangulation of a fixed 4-manifold representing spacetime, but there is also great interest in more abstract 'spin foam models', where there is no underlying manifold [6, 7, 16, 22]. Again, these models come in both Euclidean and Lorentzian versions. The Lorentzian versions are more physically realistic, but they involve extra difficulties due to the noncompactness of the Lorentz group $\mathrm{SO}_{0}(3,1)$, the solution of which forms the main topic of this paper.

\section{Evaluating RELATivistic SPIN NETWORKS}

As discussed informally in the introduction, a relativistic spin network is a graph with an assignment of a real number $p \geq 0$ to each edge. In what follows only relativistic spin networks whose underlying graph is connected are considered.

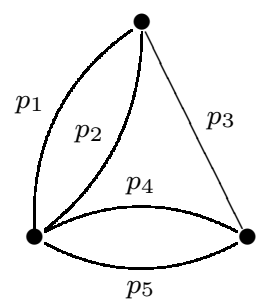

FiguRE 1. A relativistic spin network 
The idea of an evaluation is a function that gives a number for each relativistic spin network. In [11] an integral was defined which determines a number for the relativistic spin network, if it converges. The integral is based on the following kernel:

$$
K_{p}(x, y)=K_{p}(r)=\frac{\sin p r}{p \sinh r}
$$

where $x$ and $y$ are two points in three-dimensional hyperbolic space, $H$, and $r$ is the distance between them. This formula defines the function $K_{p}(r)$ for $p, r>0$, but it extends uniquely to a continuous function of $p, r \geq 0$, with $K_{p}(0)=1$ and $K_{0}(r)=r / \sinh r$.

The integral is defined as follows. First, to each vertex $v \in V$ of the graph we associate a variable $x_{v} \in H$. Each edge $e \in E$ thus has two variables, $x_{s(e)}$ and $x_{t(e)}$, associated to its endpoints. Next, to each edge $e$ of the graph we associate a factor of $K_{p}\left(x_{s(e)}, x_{t(e)}\right)$, which depends on the edge label $p$.

The idea is then to integrate the product of these factors $\prod_{E} K$ over the variables in hyperbolic space. However, since this product is invariant under the action of $\mathrm{SO}_{0}(3,1)$ as isometries of $H$, one of the integrations is redundant, and would lead to an infinite value for the integral. Thus we arbitrarily choose one vertex, say $w$, and omit the integration over the variable associated to that vertex. Let $V^{\prime}=$ $V-\{w\}$ be the remaining set of vertices, and $n$ the total number of vertices.

The integral is then given as follows:

$$
I_{w}\left(x_{w}\right)=\frac{1}{\left(2 \pi^{2}\right)^{n-1}} \int_{H^{n-1}} \prod_{E} K_{p(e)}\left(x_{e(0)}, x_{e(1)}\right) \prod_{v \in V^{\prime}} \mathrm{d} x_{v}
$$

The measure $\mathrm{d} x$ on hyperbolic space is the standard Riemannian volume measure for the unit hyperboloid. In spherical coordinates where $r$ is the distance from a fixed origin

$$
\mathrm{d} x=\sinh ^{2} r \mathrm{~d} r \mathrm{~d} \Omega,
$$

where $d \Omega$ is Lebesgue measure on the unit 2-sphere.

If the integral converges, it defines a function of the remaining variable, $x_{w}$. However the Lorentz invariance gives $I_{w}\left(x_{w}\right)=I_{w}\left(L\left(x_{w}\right)\right)$ for any $L \in \mathrm{SO}_{0}(3,1)$, so the integral is actually a constant, say $I_{w}$.

The Lorentz invariance also implies that $I_{w}$ is independent of the choice of the vertex $w$. This follows from the formula

$$
I_{w}\left(x_{w}\right)=\int_{H} I\left(x_{w}, x_{v}\right) \mathrm{d} x_{v}
$$


where the integrand is obtained by integrating over all but two variables. This function is an invariant function on $H \times H$, and therefore a function of the hyperbolic distance between its two arguments. Any such function is symmetric in its arguments. This establishes the equality $I_{w}\left(x_{w}\right)=I_{v}\left(x_{v}\right)$.

Definition 1. The evaluation of a relativistic spin network is defined as the common value of $I_{w}\left(x_{w}\right)$ for any choice of vertex $w$ and $x_{w} \in H$.

Clearly this definition only works in the cases when the integral converges. We have not yet been precise about what this means. The best situation is when the integrand in the definition is integrable, i.e., when the Lebesgue integral of its absolute value exists. In this case we say that the relativistic spin network is integrable. The results in this paper refer to the integrable case. If the network is integrable for all values of the edge labels $p$, then the graph will be called integrable, and the evaluation defines a function of these edge labels. Our first result is that this function is bounded:

Theorem 1. For an integrable graph, the relativistic spin network evaluation is bounded by a constant that is independent of the edge labels.

A more general situation is where the integral defines a generalised function, or distribution, in the $p$ variables. In this situation the relativistic spin network may not be integrable for specific values of the edge labels $p$. However, the integrand in the definition of the evaluation will become integrable after smoothing with suitable test functions in the $p$ variables. We are not going to be precise about the details of the test functions, but will confine ourselves to noting some simple examples where this phenomenon occurs. In this case, the graph will be called distributional. If the graph is not even distributional, it will be called divergent.

Some simple cases, calculated in [11], illustrate these definitions. A graph given by a single loop with one vertex on it:

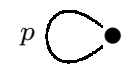

has the evaluation

$$
K_{p}(x, x)=1 .
$$

A graph with two vertices on a loop:

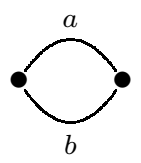


has the evaluation

$$
\frac{1}{2 \pi^{2}} \int_{H} K_{a}(x, y) K_{b}(y, x) \mathrm{d} y=\frac{2}{\pi a b} \int_{0}^{\infty} \sin a r \sin b r \mathrm{~d} r=\frac{\delta(a-b)}{a^{2}} .
$$

This is an example of a relativistic spin network that is not integrable for any values of $a$ and $b$. However the result is well defined after smoothing in $a$ and $b$, so the graph is distributional. In general this phenomenon occurs whenever the graph has a bivalent vertex.

In the simpler case of a closed network with two vertices joined with just one edge:

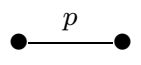

the evaluation is a divergent integral for all values of the label $p$.

$$
\frac{1}{2 \pi^{2}} \int_{H} K_{p}(x, y) \mathrm{d} y=\frac{1}{2 \pi^{2} p} \int_{0}^{\infty} \sinh r \sin p r \mathrm{~d} r .
$$

More generally, any graph with a univalent vertex is divergent.

The theta graph with two vertices and three edges:

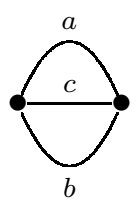

is integrable and has the evaluation

$$
\begin{aligned}
& \frac{1}{2 \pi^{2}} \int_{H} K_{a}(x, y) K_{b}(x, y) K_{c}(x, y) \mathrm{d} y=\frac{2}{\pi a b c} \int_{0}^{\infty} \frac{\sin a r \sin b r \sin c r}{\sinh r} \mathrm{~d} r \\
&=\frac{1}{4 a b c}(\tanh (\left.\frac{\pi}{2}(b+c-a)\right)+\tanh \left(\frac{\pi}{2}(c+a-b)\right) \\
&\left.+\tanh \left(\frac{\pi}{2}(a+b-c)\right)-\tanh \left(\frac{\pi}{2}(a+b+c)\right)\right)
\end{aligned}
$$

The graphs with two vertices and more than three connecting edges are also integrable and can be evaluated explicitly.

Now we can extend these examples by stating our main results on integrability. In what follows, we call the complete graph on 4 vertices the 'tetrahedron':

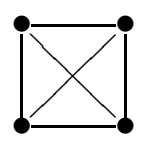


and call the complete graph on 5 vertices the ' 4 -simplex':

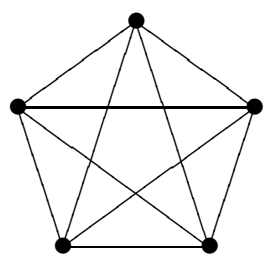

Theorem 2. The tetrahedron is an integrable graph.

Theorem 3. A graph obtained from an integrable graph by connecting an extra vertex to the existing graph by at least three extra edges is integrable. A graph obtained from an integrable graph by adding extra edges is integrable. A graph constructed by joining two disjoint integrable graphs at a vertex is integrable.
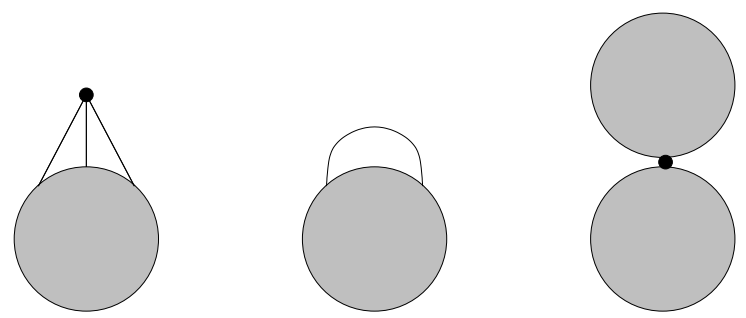

Theorem 3 allows the construction of a large class of integrable graphs. Starting with a graph which is known to be integrable, such as the theta graph or the tetrahedron, then one can construct larger integrable graphs by successively carrying out the operations described in the theorem. In particular, the 4-simplex is integrable because it is obtained by adding a vertex connected by four edges to the tetrahedron.

\section{Proof of the Main Results}

In equation (4) we defined the evaluation of a relativistic spin network as a certain integral over $n$-tuples of points in hyperbolic space. To prove Theorems 2 and 3, we need to show this integral converges. Before starting the proofs, we give an informal outline of the procedure.

Our procedure is to integrate over one point (variable) at a time, treating the remaining ones as fixed. This is justified by the theorems of Fubini and Tonelli concerning the Lebesgue integral of a function of several variables [29].

For an example of this procedure, consider the integral

$$
\int_{H} \mathrm{~d} x K_{p_{1}}\left(x, x_{1}\right) K_{p_{2}}\left(x, x_{2}\right) \cdots K_{p_{n}}\left(x, x_{n}\right)
$$


for fixed $x_{1}, \ldots, x_{n} \in H$. To prove that this integral converges we need a bound on the kernel $K$ (proved below in Lemma 11): for any $\epsilon>0$, there exists a constant $c$ (independent of $p$ ) such that

$$
\left|K_{p}(r)\right| \leq c e^{-(1-\epsilon) r}
$$

Using this, it follows that the integral is bounded by

$$
c^{n} \int_{H} \mathrm{~d} x e^{-(1-\epsilon)\left(r_{1}+\cdots+r_{n}\right)}
$$

where $r_{i}=d\left(x, x_{i}\right)$. Now suppose we can find a 'barycentre' for the points $x_{i}$, that is, a point $b \in H$ such that

$$
r:=d(x, b) \leq \frac{1}{n}\left(r_{1}+\cdots+r_{n}\right)
$$

for all $x$. Then if we work in spherical coordinates centered at $b$, we see that the integral is bounded by

$$
4 \pi c^{n} \int_{0}^{\infty} e^{-(1-\epsilon) n r} \sinh ^{2} r \mathrm{~d} r
$$

which converges for all $n \geq 3$ providing we pick $0<\epsilon<1 / 3$.

This example illustrates the importance of adding 3 or more new edges for each new vertex in the graph. To prove our main results, we now prove the above bound on the kernel $K$, construct the required barycentres, and give an improved version of the above estimate. Finally, we give a careful treatement of the tetrahedron graph.

We begin the formal proofs by bounding $K$. First note that $|\sin p r| \leq$ $p r$ so that for $p>0$

$$
\left|K_{p}(r)\right|=\frac{|\sin p r|}{p \sinh r} \leq \frac{r}{\sinh r} .
$$

This bound on $K_{p}(r)$ also holds when $r$ is zero, as long as we define $r / \sinh r$ to equal 1 when $r=0$. The right-hand side of the inequality is $K_{0}(r)$.

Proof of Theorem 1. By inequality 5, the evaluation of a relativistic spin network is bounded by the evaluation of the network with the edge label $p=0$ for each edge.

Now we give the detailed estimates needed for Theorems 2 and 3 .

Lemma 1. For any $\epsilon>0$, there exists a constant $c$ such that

$$
\left|K_{p}(r)\right| \leq c e^{-(1-\epsilon) r}
$$

for all $p \geq 0$ and $r \geq 0$. Also, $\left|K_{p}(r)\right| \leq 1$ for all $p, r$. 
Proof. Since the function $r / \sinh r$ is bounded and is asymptotic to $2 r e^{-r}$ as $r \rightarrow+\infty$, for any $\epsilon>0$ there exists $c$ with

$$
\frac{r}{\sinh r} \leq c e^{-(1-\epsilon) r} .
$$

The second part follows because $r / \sinh r$ is bounded by 1 .

Next we construct a barycentre for any finite collection of points in hyperbolic space, beginning with the case of two points.

Lemma 2. Suppose $p_{1}, p_{2} \in H$ and let $p$ be the midpoint of the geodesic from $p_{1}$ to $p_{2}$. For any point $q \in H$ we have

$$
d(p, q) \leq \frac{1}{2}\left(d\left(p_{1}, q\right)+d\left(p_{2}, q\right)\right)
$$

Proof. Using notation as in this picture:

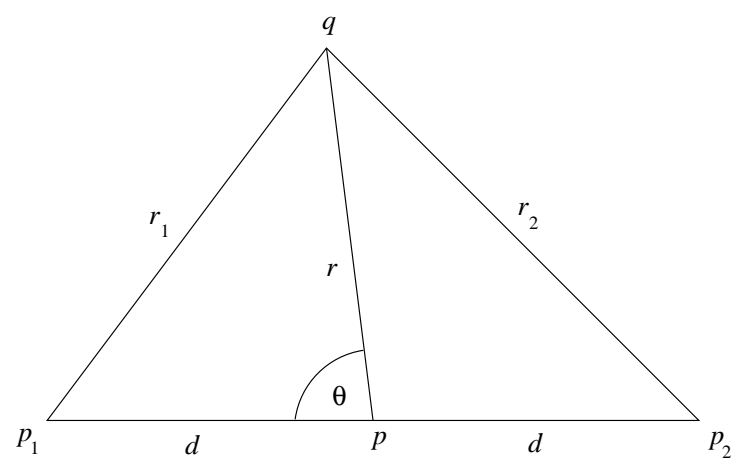

we need to show $r \leq \frac{1}{2}\left(r_{1}+r_{2}\right)$. By the law of cosines for hyperbolic trigonometry [24] we have

$$
\begin{aligned}
& \cosh d \cosh r=\cosh r_{1}+\cos \theta \sinh d \sinh r, \\
& \cosh d \cosh r=\cosh r_{2}-\cos \theta \sinh d \sinh r .
\end{aligned}
$$

Adding these equations we obtain

$$
\cosh d \cosh r=\cosh \left(\frac{r_{1}-r_{2}}{2}\right) \cosh \left(\frac{r_{1}+r_{2}}{2}\right) .
$$

By the triangle inequality we have $\left|r_{1}-r_{2}\right| \leq 2 d$, so

$$
\cosh r \leq \cosh \left(\frac{r_{1}+r_{2}}{2}\right),
$$

from which the desired result follows.

There are a variety of ways of constructing a barycentre for 3 or more points. First we give an intuitive method for 3 points, followed by an proof that a barycentre exists for any finite number of points. 
A barycentre for 3 points can be constructed by an iterative process. Begin by constructing midpoints of the geodesics between the points $p_{1}, p_{2}$ and $p_{3}$ as in this picture:

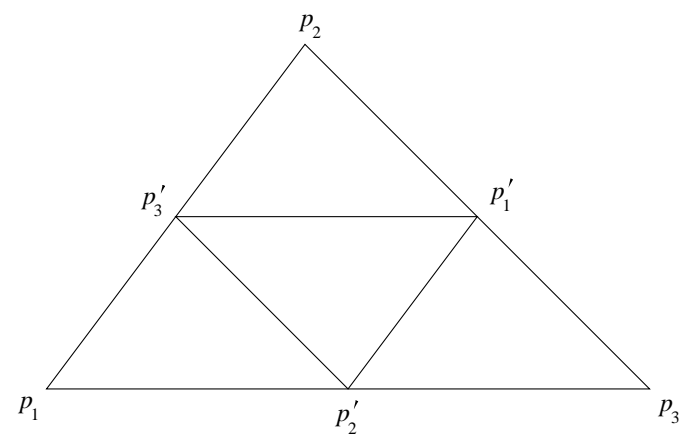

Lemma 2 implies the inequality

$$
d\left(p_{1}, q\right)+d\left(p_{2}, q\right)+d\left(p_{3}, q\right) \geq d\left(p_{1}^{\prime}, q\right)+d\left(p_{2}^{\prime}, q\right)+d\left(p_{3}^{\prime}, q\right) .
$$

Iterating this process, we obtain a sequence of nested triangles in hyperbolic space:

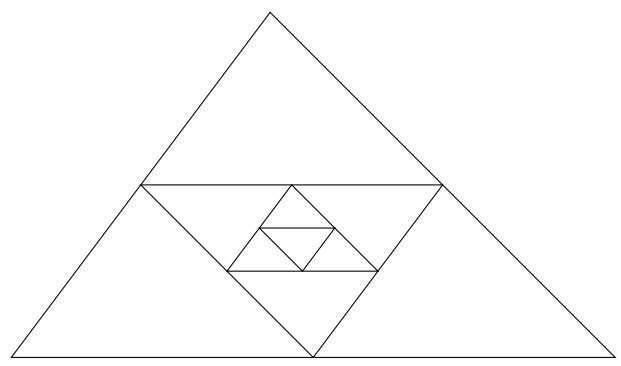

The vertices converge to a point $p$, the unique point contained in all the triangles. By repeated use of the above inequality

$$
3 d(p, q) \leq d\left(p_{1}, q\right)+d\left(p_{2}, q\right)+d\left(p_{3}, q\right),
$$

so that $p$ is a barycentre.

The following constructions work for all $n$. First, consider the case where the points lie along a straight line, i.e. a geodesic $\gamma \in H$. Then since $\gamma$ is isometric to the real line with its usual metric, the arithmetric mean of the points is defined. The next lemma shows that this is a barycentre.

Lemma 3. Suppose $\gamma \subset H$ is a geodesic, and $p_{1}, p_{2}, \ldots, p_{n} \in \gamma$. Then the arithmetic mean $p$ of the points has the property that for any point $q \in H$ we have

$$
d(p, q) \leq \frac{1}{n}\left(d\left(p_{1}, q\right)+d\left(p_{2}, q\right)+\cdots+d\left(p_{n}, q\right)\right) .
$$


Proof. This is proved by iteration. Suppose $p_{1}$ and $p_{2}$ are two points in the set which are the farthest distance apart. Then using Lemma 2, we can replace both $p_{1}$ and $p_{2}$ by the barycentre of $p_{1}$ and $p_{2}$ without increasing the quantity

$$
d\left(p_{1}, q\right)+d\left(p_{2}, q\right)+\cdots+d\left(p_{n}, q\right) .
$$

By iterating this process, all of the points in the set converge to the arithmetic mean $p$.

Now the main result on barycentres is proved.

Lemma 4. Suppose $p_{1}, p_{2}, \ldots, p_{n} \in H$. Then there exists a point $p$ such that for any point $q \in H$ we have

$$
d(p, q) \leq \frac{1}{n}\left(d\left(p_{1}, q\right)+d\left(p_{2}, q\right)+\cdots+d\left(p_{n}, q\right)\right) .
$$

Proof. This is proved by induction on $n$. The induction starts with $n=2$ by Lemma 2. Let $b$ be a barycentre for the first $n-1$ points. Then by the induction hypothesis

$$
(n-1) d(b, q)+d\left(p_{n}, q\right) \leq d\left(p_{1}, q\right)+d\left(p_{2}, q\right)+\cdots+d\left(p_{n}, q\right) .
$$

Next we find a barycentre $p$ for $n-1$ points at $b$ and 1 point at $p_{n}$. To do this, note that all these points lie on a geodesic, and so the barycentre is constructed by Lemma 3. We thus have

$$
\begin{aligned}
n d(p, q) & \leq(n-1) d(b, q)+d\left(p_{n}, q\right) \\
& \leq d\left(p_{1}, q\right)+d\left(p_{2}, q\right)+\cdots+d\left(p_{n}, q\right) .
\end{aligned}
$$

Next we prove an improved version of the estimate given at the beginning of this section. Suppose $x_{1}, \ldots, x_{n}$ are fixed in $H$ and $r_{i j}=$ $d\left(x_{i}, x_{j}\right)$.

Lemma 5. If $n \geq 3$, the integral

$$
J=\int_{H} \mathrm{~d} x\left|K_{p_{1}}\left(x, x_{1}\right) K_{p_{2}}\left(x, x_{2}\right) \cdots K_{p_{n}}\left(x, x_{n}\right)\right|
$$

converges, and for any $0<\epsilon<1 / 3$ there exists a constant $C>0$ such that for any choice of the points $x_{1}, \ldots, x_{n}$,

$$
J \leq C \exp \left(-\frac{n-2-n \epsilon}{n(n-1)} \sum_{i<j} r_{i j}\right)
$$


Proof. Define $r_{i}=d\left(x_{i}, x\right)$. Using Lemma 11 we obtain

$$
\left|K_{p_{1}}\left(x, x_{1}\right) \cdots K_{p_{n}}\left(x, x_{n}\right)\right| \leq c^{n} e^{-(1-\epsilon) \sum r_{i}} .
$$

The summation here, and in the rest of the proof of the lemma, is over values of $i$ from 1 to $n$. If we work in spherical coordinates using the barycentre of the points $x_{1}, x_{2}, \ldots, x_{n}$ as our origin, this implies

$$
J \leq 4 \pi c^{n} \int_{0}^{\infty} e^{-(1-\epsilon) \sum r_{i}} \sinh ^{2} r \mathrm{~d} r .
$$

We next break this integral over $r$ into two parts, and estimate these separately using two bounds:

$$
\sum r_{i} \geq n r
$$

from Lemma 1 , and

$$
\sum r_{i} \geq n M
$$

where

$$
M=\frac{1}{n} \min _{x} \sum r_{i}
$$

We obtain

$$
\begin{aligned}
J & \leq 4 \pi c^{n}\left[\int_{0}^{M} e^{-n(1-\epsilon) M} \sinh ^{2} r \mathrm{~d} r+\int_{M}^{\infty} e^{-n(1-\epsilon) r} \sinh ^{2} r \mathrm{~d} r\right] \\
& \leq \pi c^{n}\left[\int_{0}^{M} e^{-n(1-\epsilon) M+2 r} \mathrm{~d} r+\int_{M}^{\infty} e^{-n(1-\epsilon) r+2 r} \mathrm{~d} r\right] \\
& \leq C e^{-(n-2-n \epsilon) M}
\end{aligned}
$$

for some constant $C>0$ depending only on $\epsilon$ and $n$. Finally, the triangle inequality implies

$$
\sum r_{i} \geq \frac{1}{n-1} \sum_{i<j} r_{i j}
$$

for all choices of $x_{1}, x_{2}, \ldots, x_{n}$, so

$$
M \geq \frac{1}{n(n-1)} \sum_{i<j} r_{i j} .
$$

Proof of Theorem 3. Firstly, consider introducing an extra vertex connected to an integrable graph $\gamma$ by three or more extra edges. Lemma 5 shows that doing the integral over the extra vertex first introduces an extra multiplicative factor of $J$ in the integral of $\prod_{E}|K|$ for the graph $\gamma$. However the function $J$ is bounded so the new graph is also integrable. 
Adding an extra edge does not affect the integrability of a graph because $|K|<1$, by Lemma 1 .

Finally, for two integrable graphs joined by identifying one vertex we have that the evaluation of the resulting graph is the product of the evaluations of the two pieces, and so in particular the graph is integrable. This follows from taking the vertex where the two pieces are joined as the vertex which is not integrated over in the definition of the evaluation.

By using Lemma 5 to evaluate a bound for the integral one vertex at a time, one can actually prove that the $n$-simplex is integrable for $n \geq 5$. However to do the important cases of the tetrahedron and the 4-simplex requires a more sensitive bound at the stage where there are 3 vertices.

Proof of Theorem g. We need to show for any choice of numbers $p_{i j} \geq 0$ for $1 \leq i<j \leq 4$ and a point $x_{1} \in H$, the integral

$$
\begin{gathered}
I=\int_{H^{3}} \mathrm{~d} x_{2} \mathrm{~d} x_{3} \mathrm{~d} x_{4} \mid K_{p_{12}}\left(x_{1}, x_{2}\right) K_{p_{13}}\left(x_{1}, x_{3}\right) K_{p_{14}}\left(x_{1}, x_{4}\right) \\
K_{p_{23}}\left(x_{2}, x_{3}\right) K_{p_{24}}\left(x_{2}, x_{4}\right) K_{p_{34}}\left(x_{3}, x_{4}\right) \mid
\end{gathered}
$$

converges.

First we integrate out $x_{4}$ using Lemma 5, obtaining

$I \leq C \int_{H^{2}} \mathrm{~d} x_{2} \mathrm{~d} x_{3} e^{-\frac{1}{6}(1-3 \epsilon)\left(r_{12}+r_{13}+r_{23}\right)}\left|K_{p_{12}}\left(x_{1}, x_{2}\right) K_{p_{13}}\left(x_{1}, x_{3}\right) K_{p_{23}}\left(x_{2}, x_{3}\right)\right|$

where $r_{i j}=d\left(x_{i}, x_{j}\right)$.

Next integrate over another variable, say $x_{3}$. With

$$
L=\int_{H} \mathrm{~d} x_{3} e^{-\frac{1}{6}(1-3 \epsilon)\left(r_{13}+r_{23}\right)}\left|K_{p_{13}}\left(x_{1}, x_{3}\right) K_{p_{23}}\left(x_{2}, x_{3}\right)\right|
$$

this gives

$$
I \leq C \int_{H} \mathrm{~d} x_{2} L e^{-\frac{1}{6}(1-3 \epsilon) r_{12}}\left|K_{p_{12}}\left(x_{1}, x_{2}\right)\right| .
$$

By equation (5) we have

$$
\begin{aligned}
L & \leq \int_{H} \mathrm{~d} x_{3} \frac{r_{13} r_{23} e^{-\frac{1}{6}(1-3 \epsilon)\left(r_{13}+r_{23}\right)}}{\sinh r_{13} \sinh r_{23}} \\
& \leq \int_{H} \mathrm{~d} x_{3} \frac{\left(r_{13}+r_{23}\right)^{2} e^{-\frac{1}{6}(1-3 \epsilon)\left(r_{13}+r_{23}\right)}}{\sinh r_{13} \sinh r_{23}}
\end{aligned}
$$


To get a good bound on the integral here, we resort to a coordinate system in which two of the coordinates are

$$
k=\frac{1}{2}\left(r_{13}+r_{23}\right), \quad \ell=\frac{1}{2}\left(r_{13}-r_{23}\right),
$$

while the third is the angle $\phi$ between the plane containing $x_{1}, x_{2}, x_{3}$ and a given plane containing $x_{1}$ and $x_{2}$. The ranges of these coordinates are

$$
r / 2 \leq k<\infty, \quad-r / 2 \leq \ell \leq r / 2, \quad 0 \leq \phi<2 \pi,
$$

where we set $r=r_{12}$. Coordinates of this sort can also be defined in Euclidean space, where they are closely akin to prolate spheroidal coordinates [20], but here all the formulas are a bit different, since we are working in hyperbolic space. The main thing we need is a formula for the volume form in these coordinates,

$$
\mathrm{d} x_{3}=2 \frac{\sinh r_{13} \sinh r_{23}}{\sinh r} \mathrm{~d} k \mathrm{~d} \ell \mathrm{d} \phi
$$

which is proved in the Appendix.

Using this formula we can do the integral (7) in the $(k, \ell, \phi)$ coordinate system, obtaining

$$
L \leq 8 \int_{0}^{2 \pi} \mathrm{d} \phi \int_{r / 2}^{\infty} \mathrm{d} k \int_{-r / 2}^{r / 2} \mathrm{~d} \ell \frac{k^{2} e^{-\frac{1}{3}(1-3 \epsilon) k}}{\sinh r}
$$

or doing the integral over $\phi$ and $\ell$ and then $k$,

$$
\begin{aligned}
L & \leq \frac{16 \pi r}{\sinh r} \int_{r / 2}^{\infty} k^{2} e^{-\frac{1}{3}(1-3 \epsilon) k} \mathrm{~d} k \\
& \leq \frac{\left(A r^{3}+B\right) e^{-\frac{1}{6}(1-3 \epsilon) r}}{\sinh r}
\end{aligned}
$$

for some constants $A$ and $B$ independent of all the parameters in this problem.

We conclude the proof by using this bound on $L$ to bound the integral I. By (5) and (6) we have

$$
\begin{aligned}
I & \leq C \int_{H} \mathrm{~d} x_{2} L e^{-\frac{1}{6}(1-3 \epsilon) r}\left|K_{p_{12}}\left(x_{1}, x_{2}\right)\right| \\
& \leq 4 \pi C \int_{0}^{\infty} L r e^{-\frac{1}{6}(1-3 \epsilon) r} \sinh r \mathrm{~d} r
\end{aligned}
$$

and by (8) this gives

$$
I \leq 4 \pi C \int_{0}^{\infty} r\left(A r^{3}+B\right) e^{-\frac{1}{3}(1-3 \epsilon) r} \mathrm{~d} r .
$$

The right-hand side is finite so the proof is complete. 


\section{Remarks and Conclusions}

Theorem 3 gives a large class of integrable graphs, starting with the theta and tetrahedron graphs. However there are further examples of integrable graphs. For example, the graph in Figure 1 is also integrable, but cannot be constructed from any integrable graph by the methods of Theorem 3. Its integrability follows by applying Lemma 5 to one of the trivalent vertices.

It seems reasonable to conjecture that any 3-edge-connected graph is integrable. A 3-edge-connected graph is one that remains connected when any edge is removed or any pair of edges are removed.

It seems that the bound in Theorem 1 should be dramatically improved. Indeed, $K$ satisfies the bound $|K|<1 /(p \sinh r)$ for $r>1 / p$, thus for large $p$ one expects the evaluation to behave like $1 / p$ for each edge variable. By inspection, this is the case for the theta-graph. We conjecture that a similar bound is true for all graphs not containing edge-loops (edges with both ends at the same vertex).

It would also be interesting to consider the obvious generalization of this theory to other dimensions. For applications to quantum gravity, one would want the $(n+1)$-simplex to be an integrable graph when labelled by any representations of $\mathrm{SO}_{0}(n, 1)$ appearing in the direct integral decomposition of the space of $L^{2}$ functions on $n$-dimensional hyperbolic space.

\section{Appendix: Spheroidal Coordinates in Hyperbolic Space}

If we fix two points $x_{1}, x_{2}$ in three-dimensional hyperbolic space, and pick a hyperbolic plane containing the geodesic between these points, we can define spheroidal coordinates on hyperbolic space as follows. Given any point $x_{3}$ in hyperbolic space, its first two coordinates are

$$
k=\frac{1}{2}\left(r_{13}+r_{23}\right), \quad \ell=\frac{1}{2}\left(r_{13}-r_{23}\right),
$$

where $r_{i j}$ is the distance from $x_{i}$ to $x_{j}$. The third coordinate is the angle $\phi$ between the plane containing $x_{1}, x_{2}, x_{3}$ and the given plane containing $x_{1}$ and $x_{2}$. The ranges of these coordinates are

$$
r / 2 \leq k<\infty, \quad-r / 2 \leq \ell \leq r / 2, \quad 0 \leq \phi<2 \pi,
$$

where we set $r=r_{12}$.

In these coordinates, the volume form on hyperbolic space is given by

$$
\mathrm{d} x_{3}=2 \frac{\sinh r_{13} \sinh r_{23}}{\sinh r} \mathrm{~d} k \mathrm{~d} \ell \mathrm{d} \phi
$$


To prove this, it is easiest to consult the following picture and use the method of infinitesimals (or differential forms):

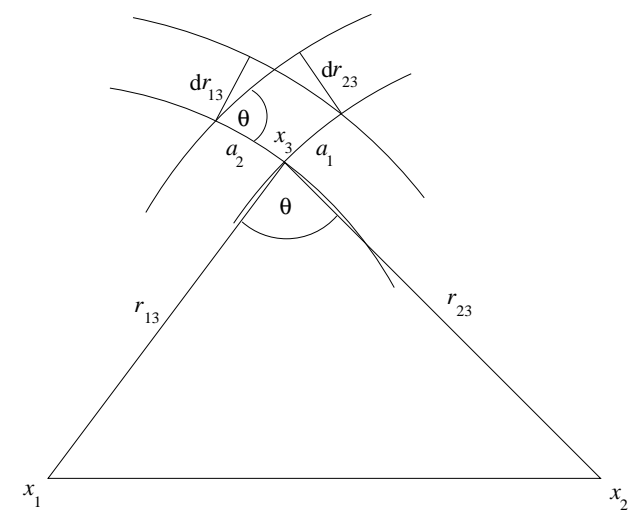

The area of the infinitesimal parallelogram formed as we vary $r_{13}$ and $r_{23}$ by amounts $\mathrm{d} r_{13}$ and $\mathrm{d} r_{23}$ is $\sin \theta a_{1} a_{2}$, where $\theta$ is the angle between the geodesics from $x_{3}$ to $x_{1}$ and $x_{2}$. Evidently $\sin \theta a_{i}=\mathrm{d} r_{i 3}$, so this area is $\mathrm{d} r_{13} \mathrm{~d} r_{23} / \sin \theta=2 \mathrm{~d} k \mathrm{~d} \ell / \sin \theta$. As we vary $\phi$ by an amount $\mathrm{d} \phi$, this parallelogram sweeps out an infinitesimal paralleliped of volume

$$
\mathrm{d} x_{3}=2 \frac{\sinh y}{\sin \theta} \mathrm{d} k \mathrm{~d} \ell \mathrm{d} \phi,
$$

where $y$ is the distance from $x_{3}$ to the geodesic between $x_{1}$ and $x_{2}$. With the help of the following picture:

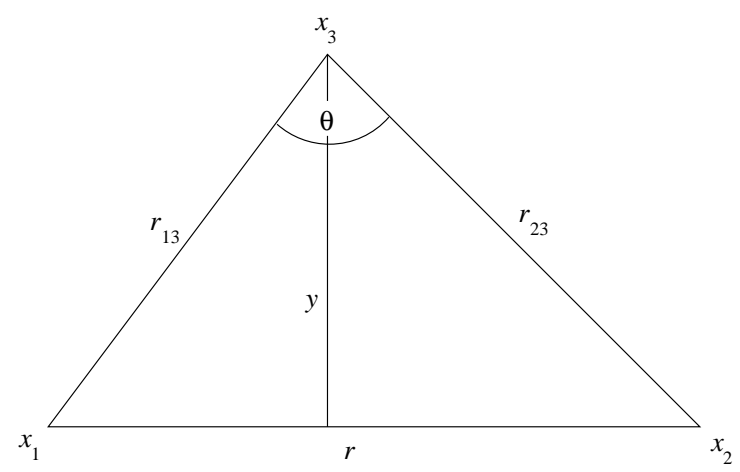

repeated use of the hyperbolic law of sines gives

$$
\sinh y=\frac{\sinh r_{13} \sinh r_{23}}{\sinh r} \sin \theta
$$

and thus

$$
\mathrm{d} x_{3}=2 \frac{\sinh r_{13} \sinh r_{23}}{\sinh r} \mathrm{~d} k \mathrm{~d} \ell \mathrm{d} \phi
$$




\section{REFERENCES}

[1] A. Ashtekar and J. Lewandowski, Quantum theory of geometry I: area operators, Class. Quantum Grav. 14 (1997), A55-A81.

[2] A. Ashtekar and J. Lewandowski, Quantum theory of geometry II: volume operators, Adv. Theor. Math. Phys. 1 (1998), 388-429.

[3] A. Ashtekar, A. Corichi and J. Zapata, Quantum theory of geometry III: noncommutativity of Riemannian structures, Class. Quantum Grav. 15 (1998), 2955-2972.

[4] J. C. Baez, Spin networks in gauge theory, Adv. Math. 117 (1996), 253-272.

[5] J. C. Baez, Spin networks in nonperturbative quantum gravity, in The Interface of Knots and Physics, ed. L. Kauffman, American Mathematical Society, Providence, Rhode Island, 1996.

[6] J. C. Baez, Spin foam models, Class. Quantum Grav. 15 (1998), 1827-1858.

[7] J. C. Baez, An introduction to spin foam models of quantum gravity and BF theory, in Geometry and Quantum Physics, eds. Helmut Gausterer and Harald Grosse, Springer, Berlin, 2000.

[8] J. C. Baez and J. W. Barrett, The quantum tetrahedron in 3 and 4 dimensions, Adv. Theor. Math. Phys. 3 (1999), 815-850.

[9] J. W. Barrett, The classical evaluation of relativistic spin networks, Adv. Theor. Math. Phys. 2 (1998) 593-600.

[10] J. W. Barrett and L. Crane, Relativistic spin networks and quantum gravity, J. Math. Phys. 39 (1998) 3296-3302.

[11] J. W. Barrett and L. Crane, A Lorentzian signature model for quantum general relativity, Class. Quantum Grav. 17 (2000), 3101-3118.

[12] J. W. Barrett, R. M. Williams. The asymptotics of an amplitude for the 4simplex, Adv. Theor. Math. Phys. 3 (1999), 209-214.

[13] L. Crane and D. Yetter, A categorical construction of 4d TQFTs, in Quantum Topology, eds. L. Kauffman and R. Baadhio, World Scientific, Singapore, 1993, pp. 120-130.

[14] L. Crane and D. Yetter, On the classical limit of the balanced state sum, preprint available as gr-qc/9712087.

[15] S. Davids, Semiclassical limits of extended Racah coefficients, J. Math. Phys. 41 (2000), 924-943

[16] R. De Pietri, L. Freidel, K. Krasnov, C. Rovelli, Barrett-Crane model from a Boulatov-Ooguri field theory over a homogeneous space, Nucl. Phys. B574 (2000), 785-806.

[17] L. Freidel and K. Krasnov, Simple spin networks as Feynman graphs, J. Math. Phys. 41 (2000) 1681-1690.

[18] L. Freidel, K. Krasnov and R. Puzio, BF description of higher-dimensional gravity theories, Adv. Theor. Math. Phys. 3 (1999), 1289-1324.

[19] B. Hasslacher and M. Perry, Spin networks are simplicial quantum gravity, Phys. Lett. B103 (1981), 21-24.

[20] P. Moon and D. E. Spencer, Field Theory Handbook: Including Coordinate Systems, Differential Equations, and Their Solutions, Springer, Berlin, 1988.

[21] R. Penrose, Angular momentum: an approach to combinatorial space-time, in Quantum Theory and Beyond, ed. T. Bastin, Cambridge U. Press, Cambridge, 1971. 
[22] A. Perez and C. Rovelli, Spin foam model for Lorentzian general relativity, Phys. Rev. D63 (2001), 041501.

[23] G. Ponzano and T. Regge, Semiclassical limit of Racah coefficients, in Spectroscopic and Group Theoretical Methods in Physics, ed. F. Bloch, NorthHolland, New York, 1968.

[24] J. G. Ratcliffe, Foundations of Hyperbolic Manifolds, Springer, Berlin, 1994.

[25] M. Reisenberger, On relativistic spin network vertices, J. Math. Phys. 40 (1999), 2046-2054.

[26] N. Reshetikhin and V. Turaev, Invariants of 3-manifolds via link polynomials and quantum groups, Invent. Math. 103 (1991), 547-597.

[27] C. Rovelli and L. Smolin, Spin networks in quantum gravity, Phys. Rev. D52 (1995), 5743-5759.

[28] C. Rovelli and L. Smolin, Discreteness of area and volume in quantum gravity, Nucl. Phys. B442 (1995), 593-622. Erratum, ibid. B456 (1995), 753.

[29] H. L. Royden, Real Analysis, Macmillan, New York, 1988.

[30] V. Turaev and O. Viro, State sum invariants of 3-manifolds and quantum $6 j$ symbols, Topology 31 (1992), 865-902.

[31] D. Yetter, Generalized Barrett-Crane vertices and invariants of embedded graphs, J. Knot Theory Ramifications 8 (1999), 815-829.

Department of Mathematics, University of California, Riverside CA 92507, USA

School of Mathematical Sciences, University Park, Nottingham NG7 2RD, UK 